Kalpa Publications in Computing
Volume 2, 2017, Pages 25-37
ICRISET2017. International Conference on Re-
search and Innovations in Science, Engineer-
ing \&Technology. Selected Papers in Computing

\title{
Solution Of Fuzzy Initial Value Problems By Fuzzy Laplace Transform
}

\author{
Komal R.Patel ${ }^{1}$ and Narendrasinh B.Desai ${ }^{2}$ \\ ${ }^{1}$ ITM Universe,Vadodara-390510, Gujarat,India. \\ ${ }^{2}$ ADIT,V.V.Nagar-388121, Gujarat, India. \\ komalpatel2121982@gmail.com, drnbdesai@yahoo.co.in
}

\begin{abstract}
In this paper we propose a fuzzy Laplace transform to solve fuzzy initial value problem under strongly generalized differentiability concept. The fuzzy Laplace transform of derivative was used to solve Nth-order fuzzy initial value problem. To illustrate applicability of proposed method we plot graphs for different values of r-level sets by using Mathematica Software.
\end{abstract}

Keywords - Fuzzy numbers, Fuzzy valued function,Hakuhara derivatives, Strongly generalized differentiability, Method of fuzzy laplace transform, Fuzzy initial value problems. 


\section{Introduction}

Fuzzy Differential equation is very much useful to solve to differential equation that occurs in field of Engineering, physical mathematics as well as mathematics. The concept of a fuzzy derivative was first introduced by Chang and Zadeh [19] followed up by Dubois and Prade[16]who used the extension principle in their approach. Other fuzzy derivative concepts were proposed by Puri and Ralescu [17] and Goetschel and Vaxman [24] as an extension of the Hukuhara derivative of multivalued functions. Kandel and Byatt[1]applied the concept of fuzzy differential equation to the analysis of fuzzy dynamical problems. The FDE and the initial value problem (Cauchy problem) were rigorously treated by Kaleva [13,14], Seikkala [20].

Two analytical methods for solving an Nth-order fuzzy linear differential equation with fuzzy initial conditions presented by Buckley and Feuring[7,8]. In 2003,O'Regan et al.[9] proved a super-linear result for fuzzy boundary value problems relying on general Schauder theorem in the metric space. Meanwhile Lakshmikantham et al.[10] investigated the solution of two-point boundary value problems associated with nonlinear fuzzy differential equation by using the extension principle. In 2008, Chen Minghao et al. [11] obtained the conclusion:two-point boundary value problems have analytic solution only on condition that the new structure and properties to the fuzzy number are given. In 2008, T.Allahviranloo et al. [22] solved Nth-order fuzzy linear differential equations.The fuzzy Laplace transforms for solving first order fuzzy differential equations under $\mathrm{H}$-differentiability have proposed by Allahviranloo and Ahmadi [2].A new method for solving fuzzy linear differential equations obtained by T. Allahviranloo, S.Abbasbandy, and S. Salahshour, A.Hakimzadeh [21]. The fuzzy Laplace transforms to solve second-order FIVPs under generalized H-differentiability applied by S.Salahshour, T.Allahviranloo [3].The fuzzy approximate solutions of second order fuzzy linear boundary value problems found by Xiaobin Guo,Dequan Shang and Xiaoquan.[25]

Strongly generalized differentiability was introduced and studied by Bede et al. $[5,6]$ the existence and uniqueness theorem of solution of Nth-order fuzzy differential equations under generalized differentiability was studied by $\mathrm{S}$ Salahshour [18].In 2015,Patel and Desai [15] find solution of variable coefficient fuzzy initial value problems by properties of Linear Transformtion. The strongly generalized derivative is defined for a larger class of fuzzy valued function than the H-derivative, and fuzzy differential equations can have solutions which have a decreasing length of their support. So we use this differentiability concept in the present paper. 
The Laplace transform method on fuzzy Nth-order derivative solves FLDEs in and corresponding fuzzy initial and boundary value problems. In this way Laplace transforms reduce the problem to an algebraic problem. The fuzzy Laplace transform also has the advantage that it solves problems directly, fuzzy Nth-order initial value problems without first determining a general solution and non homogeneous differential equations without first solving the corresponding homogeneous equation.

\section{Preliminaries}

\subsection{Fuzzy Number}

A fuzzy number is a fuzzy set like $u: R \rightarrow I=[0,1]$ which satisfies:

$1 . u$ is upper semi-continuous.

2.u is fuzzy convex i.e. $(\lambda x+(1-\lambda) y) \geq \min \{u(x), u(y)\} \forall x, y \in R, \lambda \in[0,1]$

3. $u$ is normal i.e $\exists x_{0} \in R$ for which $u\left(x_{0}\right)=1$

4.supp $u=\{x \in R \mid u(x)>0\}$ is support of $u$, and its closure $c l$ (suppu) is compact.

\section{$2.2 \mathrm{r}$-Level Sets}

Let $E$ be set of all real fuzzy numbers on $R$.The $r$-level set of fuzzy number $u \in E, 0 \leq r \leq 1$, denoted by $[u]_{r}$ is defined as

$$
[u]_{r}=\left\{\begin{array}{c}
\{x \in R \mid u(x) \geq r\} \text { if } 0 \leq r \leq 1 \\
\text { cl(suppu } \text { ifr }=0
\end{array}\right\}
$$

It is clear the $r$-level set of a fuzzy number is a closed and bounded interval $[(\underline{u}(r), \bar{u}(r)]$, where $\underline{u}(r)$ denote left-hand endpoint of $[u]_{r}$ and $\bar{u}(r)$ denote right-hand endpoint of $[u]_{r}$. Since each $y \in R$ can be regarded as a fuzzy number $\tilde{y}$ defined by

$R$ can be embedded in $E$.

$$
\tilde{y}(t)=\left\{\begin{array}{ll}
1 & \text { ift }=y \\
0 & \text { ift } \neq y
\end{array}\right\}
$$

\subsection{Parametric form of Fuzzy Number}

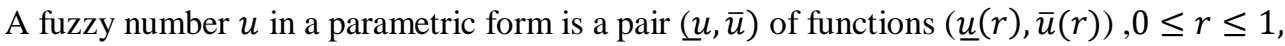
which satisfies the following requirements:

1.u $(r)$ is a bounded monotonic increasing left continuous function,

2. $\bar{u}(r)$ is a bounded monotonic decreasing left continuous function,

3. $\underline{u}(r) \leq \bar{u}(r), 0 \leq r \leq 1$.

A crisp number $\alpha$ is simply represented by $(u(r), \bar{u}(r))=\alpha, 0 \leq r \leq 1$. we recall that for $a<b<c$ which $a, b, c \in R$ the triangular fuzzy number

$u=(a, b, c)$ determine by $a, b, c$ is given such that

$u(r)=a+(b-a) r$ and $\bar{u}(r)=c-(c-b) r$ are endpoints of $r$-level sets, for all $r \in[0,1]$ 


\subsection{Properties of Fuzzy Valued Number}

For arbitrary $u=(u(r), \bar{u}(r)), v=(v(r), \bar{v}(r)), 0 \leq r \leq 1$ and arbitrary $k \in R$.

We define addition, subtraction, multiplication, scalar multiplication by $k$.

$$
\begin{aligned}
& u+v=(\underline{u}(r)+\underline{v}(r), \bar{u}(r)+\bar{v}(\mathrm{r})) \\
& u-v=(\underline{u}(r)-\bar{v}(r), \bar{u}(r)-v(\mathrm{r})) \\
& u \cdot v=(\min \{\underline{u}(r) \bar{v}(\mathrm{r}), \underline{u}(r) \underline{v}(r), \bar{u}(r) \bar{v}(\bar{r}), \bar{u}(r) \underline{v}(r)\}, \\
& \max \{\underline{u}(r) \bar{v}(\mathrm{r}), \underline{u}(r) v(r), \bar{u}(r) \bar{v}(\mathrm{r}), \bar{u}(r) \underline{v}(r)\}) \\
& k u=\left\{\begin{array}{c}
(k \underline{u}(r), k \bar{u}(r)) \quad \text { if } k \geq 0 \\
(k \bar{u}(r), k \underline{u}(r)) \text { if } k<0
\end{array}\right\}
\end{aligned}
$$

\section{Hukuhara Difference,Generlized And Strongly Generalized Differentiability}

\subsection{Hukuhara difference}

Let $x, y \in E$.If there exists $z \in E$ such that $x=y+z$, then $z$ is called the Hukuhara difference of fuzzy numbers $\mathrm{x}$ and $\mathrm{y}$, and it is denoted by $z=x \theta y$. The $\theta$ sign stands for Hukuhara-difference, and $x \theta y \neq x+(-1) y$.

\subsection{Hukuhara differential}

Let $f:(a, b) \rightarrow E$ and $t_{0} \in(a, b)$ if there exists an element $f^{\prime}\left(t_{0}\right) \in$ E such that for all $h>0$ sufficiently small,exists $f\left(t_{0}+h\right) \Theta f\left(t_{0}\right), f\left(t_{0}\right) \Theta f\left(t_{0}-h\right)$ and the limits holds(in the metric D)

$\lim _{h \rightarrow 0} \frac{\mathrm{f}\left(t_{0}+\mathrm{h}\right) \theta \mathrm{f}\left(t_{0}\right)}{h}=\lim _{h \rightarrow 0} \frac{\mathrm{f}\left(t_{0}\right) \theta \mathrm{f}\left(t_{0}-\mathrm{h}\right)}{h}=f^{\prime}\left(t_{0}\right)$

\subsection{Generalized Hukuhara Darivative}

Let $f:(a, b) \rightarrow E$ and $t_{0} \in(a, b)$ we say that $\mathrm{f}$ is (1)- differential if there exists an element $f^{\prime}\left(t_{0}\right) \in$ E such that for all $h>0$ sufficiently small, exists $f\left(t_{0}+h\right) \Theta f\left(t_{0}\right), f\left(t_{0}\right) \Theta f\left(t_{0}-h\right)$ and the limits holds(in the metric D)

$\lim _{h \rightarrow 0} \frac{\mathrm{f}\left(t_{0}+\mathrm{h}\right) \theta \mathrm{f}\left(t_{0}\right)}{h}=\lim _{h \rightarrow 0} \frac{\mathrm{f}\left(t_{0}\right) \Theta \mathrm{f}\left(t_{0}-\mathrm{h}\right)}{h}=f^{\prime}\left(t_{0}\right)$

$\mathrm{f}$ is (2)-differential if there exists an element $f^{\prime}\left(t_{0}\right) \in \mathrm{E}$ such that for all $h>0$ sufficiently small, exists $f\left(t_{0}\right) \theta f\left(t_{0}+h\right), f\left(t_{0}-h\right) \theta f\left(t_{0}\right)$ and the limits holds(in the metric D)

$\lim _{h \rightarrow 0} \frac{\mathrm{f}\left(t_{0}\right) \theta \mathrm{f}\left(t_{0}+\mathrm{h}\right)}{-h}=\lim _{h \rightarrow 0} \frac{\mathrm{f}\left(t_{0}-h\right) \Theta \mathrm{f}\left(t_{0}\right)}{-h}=f^{\prime}\left(t_{0}\right)$

If $f^{\prime}\left(t_{0}\right)$ exist in above cases then i.e. called Generalized fuzzy derivative of $f(t)$. 


\subsection{Strongly generalized differential}

Let $f:(a, b) \rightarrow E$ and $t_{0} \in(a, b)$ we say that $\mathrm{f}$ is strongly generalized differential if there exists an element $f^{\prime}\left(t_{0}\right) \in$ E such that

(i)for all $h>0$ sufficiently small, exists

$f\left(t_{0}+h\right) \Theta f\left(t_{0}\right), f\left(t_{0}\right) \Theta f\left(t_{0}-h\right)$ and the limits holds(in the metric D)

$\lim _{h \rightarrow 0} \frac{\mathrm{f}\left(t_{0}+\mathrm{h}\right) \Theta \mathrm{f}\left(t_{0}\right)}{h}=\lim _{h \rightarrow 0} \frac{\mathrm{f}\left(t_{0}\right) \Theta \mathrm{f}\left(t_{0}-\mathrm{h}\right)}{h}=f^{\prime}\left(t_{0}\right)$ or

(ii)for all $h>0$ sufficiently small, exists

$f\left(t_{0}\right) \Theta f\left(t_{0}+h\right), f\left(t_{0}-h\right) \Theta f\left(t_{0}\right)$ and the limits holds(in the metric D)

$\lim _{h \rightarrow 0} \frac{\mathrm{f}\left(t_{0}\right) \Theta \mathrm{f}\left(t_{0}+\mathrm{h}\right)}{-h}=\lim _{h \rightarrow 0} \frac{\mathrm{f}\left(t_{0}-h\right) \Theta \mathrm{f}\left(t_{0}\right)}{-h}=f^{\prime}\left(t_{0}\right)$ or

(iii)for all $h>0$ sufficiently small, exists

$f\left(t_{0}+h\right) \Theta f\left(t_{0}\right), f\left(t_{0}-h\right) \Theta f\left(t_{0}\right)$ and the limits holds(in the metric D)

$\lim _{h \rightarrow 0} \frac{\mathrm{f}\left(t_{0}+h\right) \Theta \mathrm{f}\left(t_{0}\right)}{h}=\lim _{h \rightarrow 0} \frac{\mathrm{f}\left(t_{0}-h\right) \Theta \mathrm{f}\left(t_{0}\right)}{-h}=f^{\prime}\left(t_{0}\right)$ or

(iv)for all $h>0$ sufficiently small, exists

$f\left(t_{0}\right) \Theta f\left(t_{0}+h\right), f\left(t_{0}\right) \Theta f\left(t_{0}-h\right)$ and the limits holds(in the metric D)

$\lim _{h \rightarrow 0} \frac{\mathrm{f}\left(t_{0}\right) \Theta \mathrm{f}\left(t_{0}+\mathrm{h}\right)}{-h}=\lim _{h \rightarrow 0} \frac{\mathrm{f}\left(t_{0}\right) \Theta \mathrm{f}\left(t_{0}-\mathrm{h}\right)}{h}=f^{\prime}\left(t_{0}\right)$

( $\mathrm{h}$ and $-\mathrm{h}$ at denominators mean $\frac{1}{h}$ and $\frac{1}{-h}$, respectively)

Theorem:1Let $f: R \rightarrow E$ be function and denote $f(t)=(f(t), \bar{f}(t))$ foreachr $\in[0,1]$.then

1.If $\mathrm{f}$ is (i)-differentiable, then $f(t)$ and $\bar{f}(t)$ are differentiable function and

$$
f^{\prime}(t)=\left(f^{\prime}(t), \bar{f}^{\prime}(t)\right)
$$

2.If $\mathrm{f}$ is (ii)-differentiable, then $f(t)$ and $\bar{f}(t)$ are differentiable function and $f^{\prime}(t)=\left(\bar{f}^{\prime}(t), \hat{f}^{\prime}(t)\right)$ 


\section{Method Of Fuzzy Laplace Transform}

Let $f(t)$ be continuous fuzzy-valued function. Suppose that $f(t) e^{-s t}$ improper fuzzy Riemann integrable on $[0, \infty)$ then $\int_{0}^{\infty} f(\mathrm{t}) e^{-s t} d t$ is called fuzzy Laplace transforms and is defined as

we have

$$
L[f(t)]=\int_{0}^{\infty} f(\mathrm{t}) e^{-s t} d t
$$

$$
\int_{0}^{\infty} f(\mathrm{t}) e^{-s t} d t=\left(\int_{0}^{\infty} f(\mathrm{t}) e^{-s t} d t, \int_{0}^{\infty} \bar{f}(\mathrm{t}) e^{-s t} d t\right)
$$

also by using definition of classical Laplace transform:

then we follow

$$
\begin{aligned}
& l[f(t, r)]=\int_{0}^{\infty} f(\mathrm{t}) e^{-s t} d t \\
& l[\bar{f}(t, r)]=\int_{0}^{\infty} \bar{f}(\mathrm{t}) e^{-s t} d t
\end{aligned}
$$

$L[f(t)]=(l[\mathrm{f}(t, r)], l[\bar{f}(t, r)])$

Theorem:2Let $f^{\prime}(t)$ be an integrable fuzzy-valued function, and $f(t)$ is the primitive of $f^{\prime}(t)$ on $[0, \infty)$ Then

where $f$ is (i)-differentiable

$$
L\left[f^{\prime}(t)\right]=s L[f(t)] \Theta f(0)
$$

or

$$
L\left[f^{\prime}(t)\right]=(-f(0)) \Theta(-s L[f(t)])
$$

Where $f$ is (ii) differentiable

Theorem:3 Let $f^{\prime \prime}(t)$ be integrable fuzzy-valued function, and $f(t), f^{\prime}(t)$ are primitive of $f^{\prime}(t)$, $f^{\prime \prime}(t)$ on $[0, \infty)$. Then

$L\left[f^{\prime \prime}(t)\right]=s^{2} L[f(t)] \Theta s f(0) \Theta f^{\prime}(0)$

where $f$ is (i)-differentiable and $f^{\prime}$ is (i)-differentiable or

$\left.L\left[f^{\prime \prime}(t)\right]=s^{2} L[f(t)] \Theta s f(0)-f^{\prime}(0)\right)$

where $f$ is (ii)-differentiable and $f^{\prime}$ is (ii)-differentiable or

$L\left[f^{\prime \prime}(t)\right]=\Theta\left(-s^{2}\right) L[f(t)]-s f(0)-f^{\prime}(0)$

where $f$ is (i)-differentiable and $f^{\prime}$ is (ii)-differentiable or

$L\left[f^{\prime \prime}(t)\right]=\Theta\left(-s^{2}\right) L[f(t)]-s f(0) \theta f^{\prime}(0)$

where $f$ is (ii)-differentiable and $f^{\prime}$ is (i)-differentiable.

Theorem:4 Linearity properties for FLT

Let $f(t)$ and $g(t)$ be continuous fuzzy-valued functions and $c_{1}, c_{2}$ are constants. suppose that $f(t) e^{-s t}, g(t) e^{-s t}$ are improper fuzzy Riemann-integrable on $[0, \infty)$ then

$$
L\left[c_{1} f(t)+c_{2} g(t)\right]=c_{1} L[f(t)]+c_{2} L[g(t)]
$$




\section{Application Of FIVPs In Mechanical Engineering}

Here we consider the application of fuzzy differential equation in Mechanical Engineering. The first one is The Vibrating Mass system without damping effect and second one is The displacement of Pendulum due to damping.

Example: 1 Consider the vibrating mass system. The mass $m=1$, the spring constant $k=4 \mathrm{lbs} / \mathrm{ft}$ and there is no, or negligible, damping. The forcing function is $2 \cos t$. The differential equation of motion is.

Subject to initial conditions

$$
y^{\prime \prime}+4 y=2 \text { cost }
$$

$$
y(0)=2, y^{\prime}(0)=0
$$

Consider the fuzzy initial value problem

By using Method of FLT

$$
\begin{gathered}
y^{\prime \prime}+4 y=2 \text { cost } \\
y(0)=[2 r, 4-2 r] \\
y^{\prime}(0)=[-2+2 r, 2-2 r]
\end{gathered}
$$

$$
L\left[y^{\prime \prime}\right]+4 L[y]=2 L[\cos t]
$$

By above Theorem

$$
\begin{gathered}
l[y(t, r)]=(2 r) \frac{s}{s^{2}+4}+(2 r-2) \frac{1}{s^{2}+4}+\frac{2 s}{\left(s^{2}+4\right)\left(s^{2}+1\right)} \\
l[\bar{y}(t, r)]=(4-2 r) \frac{s}{s^{2}+4}+(2-2 r) \frac{1}{s^{2}+4}+\frac{2 s}{\left(s^{2}+4\right)\left(s^{2}+1\right)}
\end{gathered}
$$

Hence the lower and upper bound of solution is as under respectively

$$
\begin{aligned}
& y(t, r)=(2 r) \operatorname{cso} 2 t+r \sin 2 t-\sin 2 t+\frac{2}{3} \cos t-\frac{2}{3} \cos 2 t \\
& \bar{y}(t, r)=(4-2 r) \cos 2 t+\sin 2 t-r \sin 2 t+\frac{2}{3} \cos t-\frac{2}{3} \cos 2 t
\end{aligned}
$$

The $y(t, r)$ and $\bar{y}(t, r)$ at $r=0,0.5,0.8,0.9,1$ are presented below in Figures.1,2,3, 4,5 respectively

$$
y(t)=y(t, 1)=\bar{y}(t, 1)=\frac{2}{3} \cos t+\frac{4}{3} \cos 2 t
$$




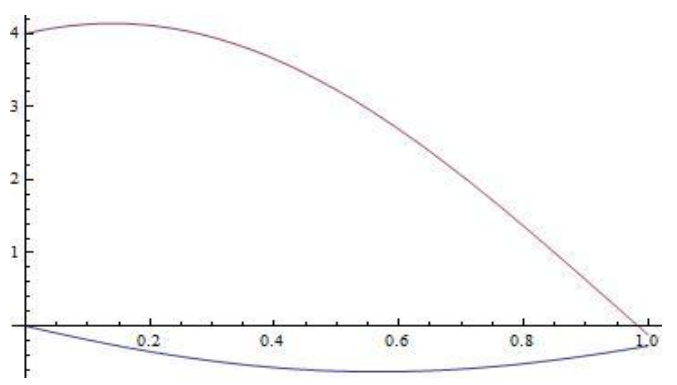

Figure $1: y(t, r)$ and $\bar{y}(t, r) a t r=0$

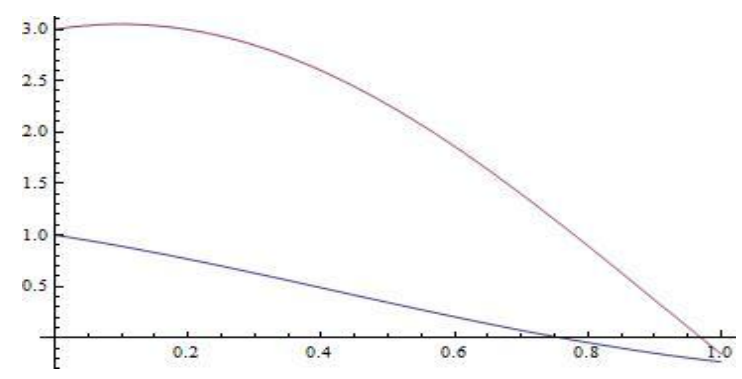

Figure 2: $y(t, r)$ and $\bar{y}(t, r)$ at $r=0.5$

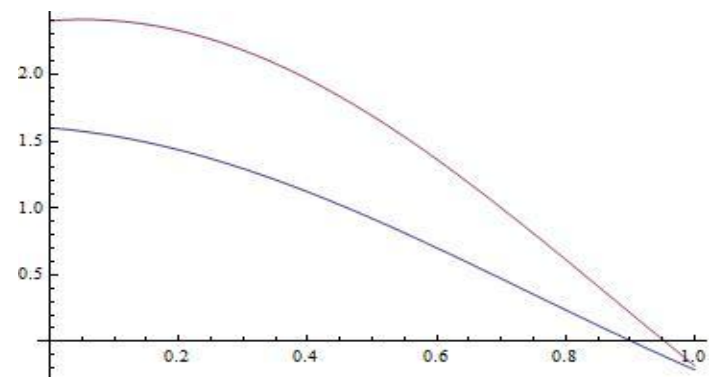

Figure 3: $y(t, r)$ and $\bar{y}(t, r)$ at $r=0.8$

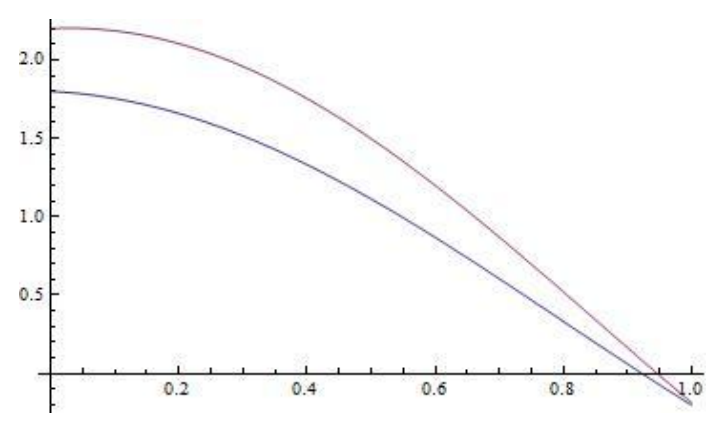

Figure 4: $y(t, r)$ and $\bar{y}(t, r)$ at $r=0.9$ 


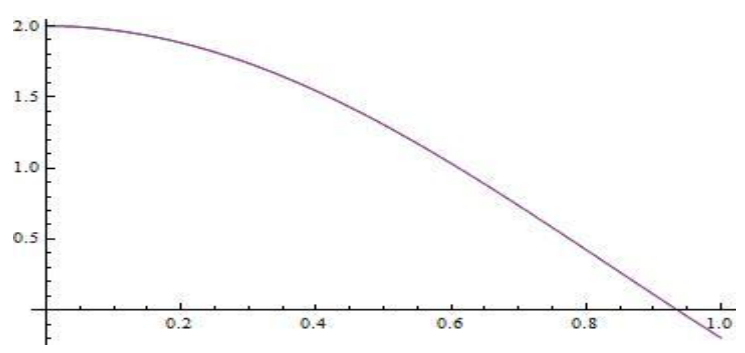

Figure 5: $y(t, r)$ and $\bar{y}(t, r)$ at $r=1$

Example:2 A Pendulum of length $\mathrm{L}=\frac{8}{5} f t$ is subject to resistive force $F_{R}=\frac{32}{5} \frac{d \theta}{d t}$ due to damping. Determine displacement function. If $\theta(0)=1, \theta^{\prime}(0)=2$.

The D.E is

$\frac{8}{5} \frac{d^{2} \theta}{d t^{2}}+\frac{32}{5} \frac{d \theta}{d t}+32 \theta=0 \quad$ With initial conditions

$$
\theta(0)=1, \theta^{\prime}(0)=2
$$

By simplifying and converting them to Fuzzy D.E

The equation become

$\frac{d^{2} \theta}{d t^{2}}+4 \frac{d \theta}{d t}+20 \theta=0$ With initial condition

$$
\theta(0)=[r, 2-r], \theta^{\prime}(0)=[1+r, 3-r]
$$

By using Method of FLT

By above Theorem

$$
L\left[\theta^{\prime \prime}\right]+4 L\left[\theta^{\prime}\right]+20 L[\theta]=L[0]
$$

$$
\begin{aligned}
& l[\theta(t, r)]=r \frac{s+2}{(s+2)^{2}+16}+\left(\frac{3 r+1}{4}\right) \frac{1}{(s+2)^{2}+16} \\
& l[\bar{\theta}(t, r)]=(2-r) \frac{s+2}{(s+2)^{2}+16}+\left(\frac{7-3 r}{4}\right) \frac{1}{(s+2)^{2}+16}
\end{aligned}
$$

Hence the lower and upper bound of solution is as under respectively

$$
\begin{aligned}
\underline{\theta}(t, r)=r e^{-2 t} \cos 4 t+\left(\frac{3 r+1}{4}\right) e^{-2 t} \sin 4 t \\
\bar{\theta}(t, r)=(2-r) e^{-2 t} \cos 4 t+\left(\frac{7-3 r}{4}\right) e^{-2 t} \sin 4 t
\end{aligned}
$$

The $\underline{\theta}(t, r)$ and $\bar{\theta}(t, r)$ at $r=0,0.5,0.8,0.9,1$ are presented below in Figurs.6,7,8, 9,10 respectively

$$
\theta(t)=\underline{\theta}(t, 1)=\bar{\theta}(t, 1)=e^{-2 t}(\cos 4 t+\sin 4 t)
$$




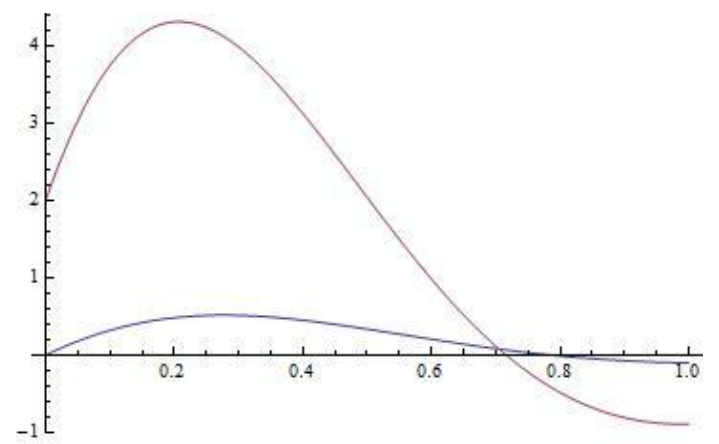

Figure 6: $\underline{\theta}(t, r)$ and $\bar{\theta}(t, r) \operatorname{atr}=0$

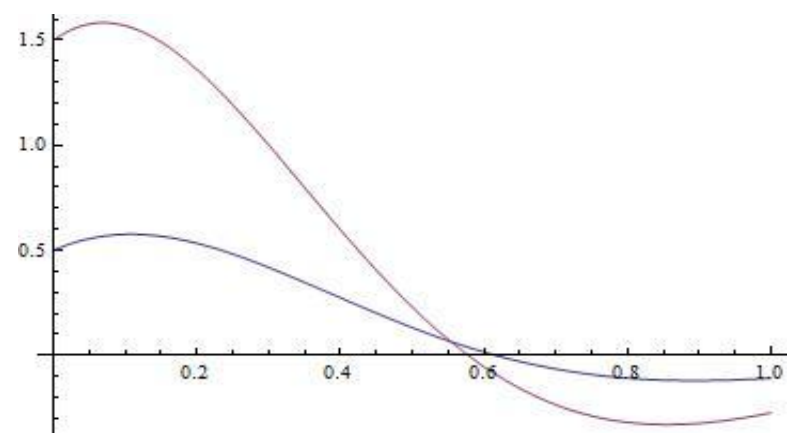

Figure 7: $\underline{\theta}(t, r)$ and $\bar{\theta}(t, r)$ at $r=0.5$

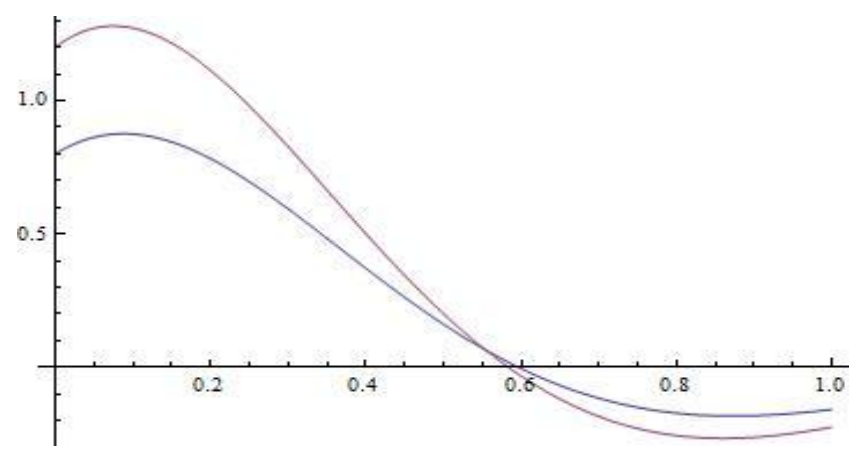

Figure 8: $\underline{\theta}(t, r)$ and $\bar{\theta}(t, r)$ at $r=0.8$ 


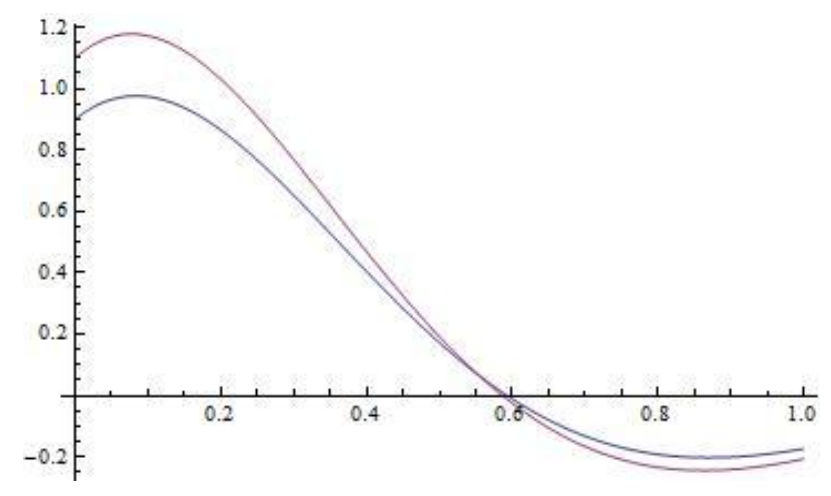

Figure 9: $\underline{\theta}(\mathrm{t}, \mathrm{r})$ and $\bar{\theta}(\mathrm{t}, \mathrm{r})$ at $\mathrm{r}=0.9$

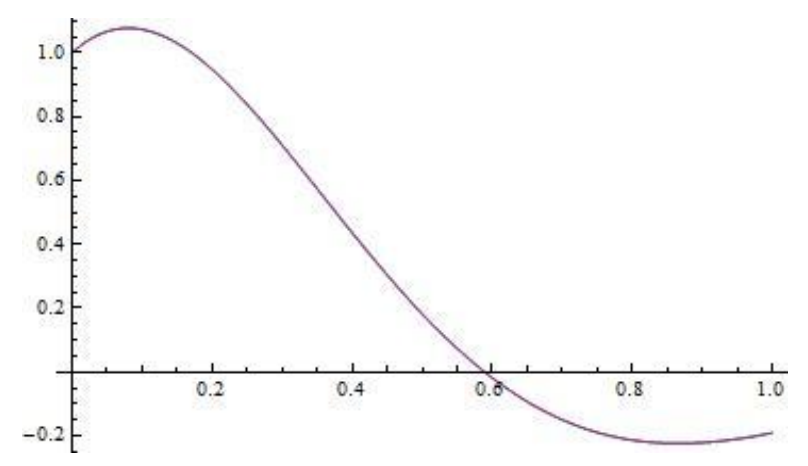

Figure 10: $\underline{\theta}(t, r)$ and $\bar{\theta}(t, r)$ at $r=1$

\section{Result and Discussion}

From above examples we see that the solution of FIVP is depends on the derivative i.e.(1)-differentiable or (2)-differentiable. Thus as in above examples, the solution can be adequately chosen among four cases of the strongly generalize differentiability. On the other hand, in this new procedure unicity of the solution is lost because we have four possibilities, but it is expected situation in the fuzzy context. In all the above examples, for different values of $r$ graphs for lower and upper bounds are different whereas at $r=1$ upper bound and lower bounds are sameand both the graphs are coincide. 


\section{Conclusion}

In this paper, the Laplace transform method provided solutions to Nth- order fuzzy initial value problem but here by sake of simplicity we have considered second order FIVPs by using the strongly generalize differentiability concept. The efficiency of Method was described by solving some application based examples. 


\section{References}

[1] A. Kandel and W. J. Byatt. (1978.)., "Fuzzy differential equations," . in Proceedings of the International Conference on Cybernetics and Society, , pp. 1213-1216, Tokyo, Japan, .

[2] Ahmadi., T. A. (2010.). Fuzzy laplace transforms. . Soft Computing, , 14(3):235-243, .

[3] Allahviranloo., S. S. (2013.). Applications of fuzzy laplace transforms. . Soft computing, , 17(1):145-158, .

[4] B, B. (2006). A note on "two-point boundary value problems associated with non-linear fuzzy differential equations". Fuzzy Sets Syst , 157:986-989.

[5] Bede B, G. S. (2005). Generalizations of the differentiability of fuzzy number valued functions with applications to fuzzy differentia lequation. Fuzzy Sets Syst, 151:581-599.

[6] BedeB, S. (2012). Generalized differentiability of fuzzy-valued functions. Fuzzy Sets Syst. , doi:10.1016/j.fss.2012.10.003.

[7] Buckley JJ, F. T. (2000). Fuzzy differential equations. Fuzzy Sets Syst, 110:43-54.

[8] Buckley JJ, F. T. (2001). Fuzzy initial value problem for Nth-order linear differential equation. Fuzzy Sets Syst, 121:247-255.

[9] Donal O'Regan, V. L. (2003). Initial and boundary value problems for fuzzy differential equations. Nonlinear Analysis: Theory, Methods \& Applications, , 54(3):405-415,.

[10] Lakshmikantham, V., \& Nieto, J. (2003). Differential equations in metric spaces:an introduction and an application to fuzzy differential equations. Dynamics of , A, vol. 10, no. 6, pp. 991-1000.

[11] Minghao Chen, C. W. ( 2008.). On fuzzy boundary value problems. . Information Sciences, , 178(7):1877-1892,.

[12] N.B.Desai. (1997). Fuzzy modeling and its application. M.Phil Dessertation .

[13] O, K. (1987). Fuzzy differential equations. Fuzzy Sets Syst. , 24:301-317.

[14] O, K. (1990). The Cauchy problem for fuzzy differential equations. . Fuzzy Sets Syst , 35:389-396.

[15] Patel K. R, D. N. (December 2015). Solution of fuzzy initial value problems. ADIT Journal of Engineering, Vol.12,No.1,53-57.

[16] Prade., D. D. (1982.). Towards fuzzy differential calculus part 3: Differentiation. Fuzzy sets and systems, , 8(3):225233, .

[17] Ralescu, M. L. (1983). Differential of fuzzy functions. Journal of Mathematical Analysis and Applications , 552$558,91(2)$.

[18] S Salahshour., S. (2011). Nth-order fuzzy differential equations under generalized differentiability. . Journal of Fuzzy Set Valued Analysis .

[19] S.L. Chang, L. Z. ( 1972). On fuzzy mapping and control,. IEEE Transactions on Systems Man Cybernetics. , (2) $330-340$.

[20] Seikkala, S. (1987). On the Fuzzy Initial Value Problem,. Fuzzy Sets Systems, , 319-330.

[21] T. Allahviranloo - S. Abbasbandy, S. S. (2011). A new method for solving fuzzy linear differential equations. Springer-Verlag , $92: 181-197$.

[22] Tofigh Allahviranloo, E. A. (86(4):730-742, 2009.). A method for solving $\mathrm{n}$ th order fuzzy linear differential equations. . International Journal of Computer Mathematics, , 86(4):730-742, 2009.

[23] Tofigh Allahviranloo, E. A. ( 2008). Nth-order fuzzy linear differential equations. Information sciences, . , 178(5):1309-1324,.

[24] Voxman, R. G. (1986). Elementary fuzzy calculus. Fuzzy sets and systems , 31-43,18(1).

[25] Xiaobin Guo, D. S.-o. (2013). Fuzzy approximate solutions of second-order fuzzy linear boundary value problems. Boundary Value Problems, , 212:1-17.

[26] Zadeh, L. A. (1975). The concept of linguistic variables and its application to approximate reasoning. Information sciences , 199-249,8(3). 